\title{
Regiões homogêneas e funções de distribuição de probabilidade da precipitação pluvial no Estado de Táchira, Venezuela
}

\author{
Gustavo Bastos Lyra(1), Beatriz Ibet Lozada Garcia( ${ }^{(2)}$, Sônia Maria De Stefano Piedade ${ }^{(1)}$, \\ Gilberto Chohaku Sediyama ${ }^{(3)}$ e Paulo César Sentelhas ${ }^{(1)}$
}

\begin{abstract}
(1)Universidade de São Paulo, Escola Superior de Agricultura Luiz de Queiroz, Dep. de Ciências Exatas, Caixa Postal 9, CEP 13418-900 Piracicaba, SP. E-mail: gblyra@gmail.com, soniamsp@esalq.usp.br, pcsentel@esalq.usp.br (2)Instituto Nacional de Investigaciones Agrícolas, CIAE - Táchira, Carretera via Delicias, Hachienda Bramón, Táchira, Venezuela. E-mail: blozada@inia.gov.ve (3)Universidade Federal de Viçosa, Dep. de Engenharia Agrícola, CEP 36570-000 Viçosa, MG. E-mail: g.sediyama@ufv.br
\end{abstract}

Resumo - O objetivo deste trabalho foi determinar regiões homogêneas baseadas na sazonalidade da precipitação pluvial mensal e a distribuição de probabilidade que melhor se ajusta à precipitação dessas regiões no Estado de Táchira, Venezuela. Utilizaram-se valores da precipitação mensal de 25 estações climatológicas, que apresentam séries entre 24 e 62 anos. Aplicou-se o método de Ward no agrupamento dos meses com precipitação pluvial mensal similar e também no das localidades com precipitação similar (regiões homogêneas). Avaliaram-se os ajustes das funções de densidade exponencial, gama, Gumbel, normal, log-normal a três parâmetros, e Weibull aos dados observados de precipitação mensal. A variação sazonal da precipitação no Estado de Táchira apresenta três períodos estatisticamente definidos como: seco, transição e úmido. Os períodos seco e úmido apresentam quatro regiões homogêneas de precipitação mensal similar e o de transição três. No período seco, a distribuição de probabilidade recomendada para as estimativas mensais é a exponencial, com exceção da região homogênea com os maiores valores de precipitação pluvial do período, onde a gama se sobressai. No período chuvoso, em todas as regiões homogêneas, a distribuição normal predomina, com exceção de agosto, em que a gama prevalece. Já nos meses de transição, destacam-se as distribuições gama, em abril, e normal, em novembro.

Termos para indexação: chuva, funções densidade de probabilidade, análise de agrupamento, Ward.

\section{Homogeneous regions and probability density functions of pluvial precipitation in Táchira State, Venezuela}

\begin{abstract}
The objective of this work was to determine regions of monthly pluvial precipitation based on the seasonal and the probability distribution that best fit to precipitation of those areas in the State of Táchira, Venezuela. Long-term series of 24 throughout 62 years of monthly precipitation data of 25 climatological stations were used. The Ward's clustering methods of analyses was used to group the months with similar monthly pluvial precipitation and also the climatological locations with similar precipitation (homogeneous regions). The adjust of the probability density functions exponential, Gamma, Gumbel, normal, log-normal were evaluated with three parameters as well as Weibull distributions in order to observe monthly precipitation data. The precipitation seasonal variation at the State of Táchira presents three periods statistically defined as: dry, transition, and wet. For the dry and wet periods, four homogeneous regions of locations with similar monthly precipitation could be identified and, in the transition period, three regions. In the dry period, the recommended probability distribution is the exponential, except for the homogeneous regions with the largest rainfall values during that period, when Gamma distribution is better. In the wet period, in all regions, normal distribution prevails, except for August, when Gamma prevails. As far as transition periods are concerned, Gamma distributions are better in April and normal distribution, in November.
\end{abstract}

Index terms: rainfall, probability density functions, cluster analysis, Ward.

\section{Introdução}

O Estado de Táchira está localizado na região sulocidental da Venezuela, na Cordilheira dos Andes e possui uma extensão territorial de $11.100 \mathrm{~km}^{2}$. O clima da região é influenciado pelos Andes que atuam como barreira orográfica entre as vertentes "llanera" e "lacustre".
Na vertente "llanera”, as baixas pressões equatoriais predominam, ao passo que na "lacustre" prevalece a ação dos ventos alísios de nordeste. Duas estações climáticas bem definidas são observadas no Estado: a seca, que vai do final de novembro a abril, caracterizando-se pela entrada da alta pressão do Atlântico, aliada ao deslocamento da zona de 
convergência intertropical (ZCIT) para o Brasil, dificultando movimentos convectivos e, dessa forma, o desenvolvimento de nuvens; e a chuvosa, que corresponde ao período de maio ao início de novembro, originada por áreas de baixa pressão atmosférica associadas à circulação ciclônica mínima, resultando em nuvens convectivas e chuvas fortes (Uzcategui \& González, 2000; D’Ambrosio, 2003).

Táchira é considerado um estado produtor de água, tanto para consumo humano quanto para atividades agrícolas e industriais. Entretanto, por sua variada topografia, as chuvas diferem consideravelmente entre suas regiões, o que dificulta o prognóstico efetivo desse recurso. Na vertente "llanera", a chuva apresenta regime unimodal e sua altura anual varia entre 1.120 e $4.000 \mathrm{~mm}$ na bacia média do Rio Uribante, enquanto na "lacustrina", o regime é bimodal, variando de 750 a 2.700 mm. D’Ambrosio (2003) cita que a variabilidade espaço-temporal característica do país é mais acentuada nas regiões montanhosas, sendo as chuvas na base dos Andes mais abundantes que no topo da cordilheira.

Em condições de diversidade da distribuição espaçotemporal da precipitação pluviométrica, escassez de estações climatológicas e necessidade de séries longas de dados em análises climatológicas, é recomendado identificar regiões similares de precipitação. Unal et al. (2003) observam que trabalhos na área climatológica têm empregado regras subjetivas para definir regiões similares, sugerindo como alternativa a utilização da análise de agrupamento (Cluster), por ser um algoritmo estatístico efetivo, que pode utilizar elementos meteorológicos observados. A análise de agrupamento é dividida em dois métodos principais: hierárquicos e não hierárquicos, e o método hierárquico de Ward é largamente utilizado em estudos climatológicos com resultados satisfatórios (Chessa et al., 1999).

O aproveitamento dos recursos hídricos de forma adequada requer o conhecimento das precipitações capazes de ocorrer em uma localidade, sendo isto possível por meio do uso das funções de probabilidade (Catalunha et al., 2002). Diversas são as teorias de probabilidade existente, não havendo uma adequada que justifique o uso de determinada distribuição (Back, 2001). Em virtude da complexidade dos processos envolvidos na variação temporal da precipitação pluviométrica, essas funções são selecionadas de acordo com o critério de melhor ajuste com séries históricas, facilidade de estimativa de seus parâmetros e flexibilidade computacional (Duan et al., 1998). Na sua maioria, as funções densidade de probabilidade apresentam de um a três parâmetros, sendo que as funções multiparâmetros geralmente mostram ajuste superior à precipitação, quando comparadas às de parâmetro único.

O objetivo deste trabalho foi determinar regiões homogêneas, de acordo com a sazonalidade da precipitação mensal e a distribuição de probabilidade que melhor se ajusta a essas regiões no Estado de Táchira, Venezuela.

\section{Material e Métodos}

Foram utilizados valores da precipitação pluvial mensal de 25 estações climatológicas do Estado de Táchira, Venezuela, obtidas pelo Ministerio del Ambiente y los Recursos Naturales (MARN) e Instituto Nacional de Investigaciones Agrícolas (INIA). As estações apresentam séries entre 24 e 62 anos.

No agrupamento dos meses com média da precipitação mensal similar, aplicou-se a análise de agrupamento (Cluster). Utilizou-se o método hierárquico aglomerativo de Ward (1963), tendo como medida de dissimilaridade a distância euclidiana (Everitt \& Dunn, 1991):

$d_{e}=\left[\sum_{j=1}^{n}\left(P_{p, j}-P_{k, j}\right)^{2}\right]^{0,5}$

em que $\mathrm{d}_{\mathrm{e}}$ é a distância euclidiana; e $\mathrm{P}_{\mathrm{p}, \mathrm{j}}$ e $\mathrm{P}_{\mathrm{k}, \mathrm{j}}$ são as variáveis quantitativas j dos indivíduos p e $k$, respectivamente.

O método de Ward forma grupos, minimizando a dissimilaridade, ou minimizando o total das somas de quadrados dentro de grupos, também conhecida como soma de quadrados dos desvios (SQD). Em cada etapa do procedimento, são formados grupos, de tal maneira que a solução resultante tenha o menor SQD dentro de grupos. Nessas etapas, são consideradas as uniões de todos os possíveis pares de grupos e, os dois que resultam em menor aumento de SQD são agrupados até que todos os grupos formem um único, reunindo todos os indivíduos (Everitt \& Dunn, 1991).

Valores médios da precipitação mensal foram organizados na forma de matriz $P_{n \times p}$, em que o elemento $P_{i j}$ representava o valor da i-ésima variável (localidade) do j-ésimo indivíduo (mês). Dessa forma, cada vetor linha representava uma localidade e cada vetor coluna um mês.

Depois do agrupamento dos meses com precipitação similar, aplicou-se novamente o método de Ward para 
determinar, dentro de cada um dos períodos mensais definidos, as regiões homogêneas de localidades com similaridade da precipitação. Nesse caso, a matriz $P_{n \times p}$ foi organizada de forma que os indivíduos representavam as localidades e os meses agrupados (períodos), as variáveis.

As distribuições de probabilidade ajustadas aos valores da precipitação mensal foram: exponencial (EX), gama (GA), Gumbel (GU), normal (N), log-normal (LN) a três parâmetros e Weibull (W). As distribuições foram ajustadas utilizando o software Estatística Aplicada a Climatologia (EClima), desenvolvido no Dep. de Engenharia Agrícola da Universidade Federal de Viçosa (DEA/UFV).

A distribuição exponencial geralmente se ajusta bem a dados que apresentam forte assimetria, como histogramas em forma de "J" invertido (Thom, 1958). Sua função densidade de probabilidade [f(x)] é expressa da seguinte forma:

$f(x)= \begin{cases}\lambda e^{-\lambda x} & x>0 \\ 0 & x<0\end{cases}$

e sua função cumulativa de probabilidade $[\mathrm{F}(\mathrm{x})]$ é dada pela expressão:

$F(x)=\int_{0}^{\infty} f(x) d x=1-e^{-\lambda x}$

em que x é a variável aleatória, que, neste caso, foi a precipitação pluvial mensal, e o parâmetro $\lambda$ é o inverso da média.

Para totais de chuva de períodos mensais ou menores, a distribuição gama de probabilidade tem sido uma das mais utilizadas (Assis et al., 1996; Silva \& Assad, 1998). Thom (1958) comenta que a distribuição gama a dois parâmetros é um caso particular da distribuição de Pearson do Tipo III, na qual o parâmetro local é zero, sendo sua função densidade de probabilidade expressa da seguinte forma:

$f(x)=\frac{1}{\beta^{\alpha} \Gamma(\alpha)} x^{\alpha-1} e^{-\frac{x}{\beta}}$ para $0<x<\infty$

com $\beta, \alpha, \Gamma(\alpha)>0$ e $\mathrm{f}(\mathrm{x})=0$ para $\mathrm{x}<0$, sendo $\beta$ o parâmetro de escala, $\alpha$ o parâmetro de forma, e $\Gamma(\alpha)$ a função gama incompleta do parâmetro $\alpha$, definida por Thom (1958):

$\Gamma(\alpha)=\int_{0}^{\infty} \mathrm{t}^{\alpha-1} \mathrm{e}^{\mathrm{t}} \mathrm{dt}$

em que $t=-x / \beta$.
A distribuição gama tem como função de distribuição acumulada:

$\mathrm{F}(\mathrm{x})=\frac{1}{\beta^{\alpha} \Gamma(\alpha)} \int_{0}^{\mathrm{x}} \mathrm{x}^{\alpha-1} \mathrm{e}^{\frac{\mathrm{x}}{\beta}} \mathrm{dx}$

A distribuição Gumbel é utilizada na análise de eventos climatológicos extremos, servindo de subsídio para projetos de engenharia agrícola (Assis et al., 1996). Uma das formas de apresentar sua função densidade de probabilidade é:

$f(x)=\frac{1}{\beta} e^{-\left(\frac{x-\alpha}{\beta}\right)}-e^{-e^{-\left(\frac{x-\alpha}{\beta}\right)}}$

em que $\alpha$ é o parâmetro posição e $\beta$ é o parâmetro de escala.

Sua função de distribuição acumulada é dada pela equação:

$\mathrm{F}(\mathrm{x})=\mathrm{e}^{-\mathrm{e}^{ \pm\left(\frac{\mathrm{x}-\alpha}{\beta}\right)}}$

O sinal \pm , no segundo expoente, refere-se aos valores extremos máximo (sinal negativo) e mínimo (sinal positivo).

A distribuição de probabilidade normal, denominada também de curva de Gauss ou curval normal, é a distribuição contínua mais utilizada (Hastings \& Peacock, 1975). A distribuição normal apresenta dois parâmetros e sua função densidade de probabilidade tem a seguinte forma:

$f(x)=\frac{1}{\sigma \sqrt{2 \pi}} e^{\left(-\frac{(x-\mu)^{2}}{2 \sigma^{2}}\right)}$

em que $\mu$ é a média e $\sigma$, o desvio-padrão.

A probabilidade acumulada de ocorrência de totais de precipitação pode ser calculada pela integração da equação 9:

$\mathrm{F}(\mathrm{x})=\frac{1}{\sigma \sqrt{2 \pi}} \int_{-\infty}^{\mathrm{x}} \mathrm{e}^{\left(-\frac{(\mathrm{x}-\mu)^{2}}{2 \sigma^{2}}\right)} \mathrm{dx}$

A função de distribuição acumulada da normal não possui solução analítica, podendo ser resolvida por métodos de integração. Assim utiliza-se a transformação $Z=(x-\mu) / \sigma$, em que $Z$ apresenta distribuição normal com média igual a zero e desvio-padrão um $[\mathrm{N}(0,1)]$. 
A variável Z é conhecida como variável reduzida. Reescrevendo a equação 10, tem-se:

$\mathrm{F}(\mathrm{Z})=\frac{1}{\sqrt{2 \pi}} \int_{-\infty}^{\mathrm{Z}} \mathrm{e}^{\left(-\frac{\mathrm{Z}^{2}}{2}\right)} \mathrm{dZ}$

Mello et al. (1994) citam que a distribuição de probabilidade log-normal é muito utilizada em hidrologia e hidroclimatologia. Essa distribuição considera que os logaritmos das variáveis aleatórias seguem uma distribuição normal (Frizzone, 1979). A função densidade de probabilidade da distribuição log-normal a três parâmetros é dada pela seguinte equação:

$f(x)=\frac{1}{(x-a) \sigma \sqrt{2 \pi}} e^{\left(-\frac{[\ln (x-a)-\mu]^{2}}{2 \sigma^{2}}\right)}$

em que $\mu$ é a média dos logaritmos da variável, $\sigma$ é o desvio-padrão dos logaritmos da variável e a é o limite inferior da amostra.

A probabilidade de que uma variável $\mathrm{x}$ assuma valores entre a e b é estimada pela sua função de distribuição acumulada, usando a relação:

$\mathrm{F}(\mathrm{a} \leq \mathrm{x} \leq \mathrm{b})=\int_{\mathrm{a}}^{\mathrm{b}} \frac{1}{(\mathrm{x}-\mathrm{a}) \sigma \sqrt{2 \pi}} \mathrm{e}^{\left(-\frac{[\ln (\mathrm{x}-\mathrm{a})-\mu]^{2}}{2 \sigma^{2}}\right)} \mathrm{dx}$

A distribuição Weibull pode ser utilizada para eventos extremos, principalmente em estudos hidrológicos, assim como a distribuição Gumbel (Duan et al., 1998). A função densidade de probabilidade da distribuição Weibull é dada por:

$\mathrm{f}(\mathrm{x})=\frac{\gamma}{\beta}\left(\frac{\mathrm{x}-\alpha}{\beta}\right)^{\gamma-1} \mathrm{e}^{-\left(\frac{\mathrm{x}-\alpha}{\beta}\right)^{\gamma}}$

Sua função de distribuição acumulada é do tipo:

$F(x)=\int_{0}^{\infty} f(x) d x=1-e^{-\left(\frac{x-\alpha}{\beta}\right)^{\nu}}$

Os parâmetros das distribuições gama, Gumbel e Weibull foram estimados pelo método da máxima verossimilhança, conforme descrito por Assis et al. (1996), Ali et al. (2000) e Catalunha et al. (2002). Utilizou-se o método da máxima verossimilhança por apresentar melhores estimativas dos parâmetros das distribuições em comparação com o método dos momentos, como citado por Assis et al. (1996), Silva \& Assad (1998) e Back (2001).
Devido à utilização da função logarítmica no cálculo dos parâmetros de algumas distribuições, meses com total de precipitação igual a zero foram substituídos por 0,1 mm, como sugerido por Ribeiro \& Lunardi (1997).

A seleção da função densidade de probabilidade que melhor se ajustou aos valores de precipitação se baseou no teste de qui-quadrado $\left(\chi^{2}\right)$, no nível de significância de $5 \%$, visto ser este mais rigoroso que o teste de Kolmogorov-Smirnov (Back, 2001; Catalunha et al., 2002). Admitiu-se que a hipótese de nulidade (Ho) é a hipótese de que os dados provêm de uma população que segue a distribuição teórica $\mathrm{F}(\mathrm{x})$ testada com seus parâmetros determinados em função dos dados amostrais (Assis et al., 1996). No caso de rejeição de Ho, isso significa que a distribuição avaliada não se ajusta aos dados observados, e o termo significativo foi utilizado para definir rejeição de Ho (Cargnelluti Filho et al., 2004).

\section{Resultados e Discussão}

Pela análise de agrupamento, observaram-se dois períodos bem diferenciados para chuva mensal, sendo um correspondente aos meses secos (janeiro, fevereiro, março e dezembro) e outro formado pelos úmidos (de abril a novembro) (Figura 1). Essa divisão coincide com as características sazonais das chuvas no Estado. O período úmido apresenta ainda uma subdivisão que separa os meses de transição (abril e novembro), no início e final desse período, daqueles totalmente úmidos (maio, junho, julho, agosto, setembro e outubro). Dessa forma, definem-se estatisticamente três períodos denominados: seco (S), transição (T) e úmido (U). A decisão do corte transversal do dendrograma para definição do número de períodos foi baseada na sazonalidade da chuva mensal e na distância de ligação entre os grupos.

Os períodos definidos estão relacionados com mecanismos de circulação de grande escala, sendo que o período seco é originado pelos alísios de nordeste que produzem subsidência das massas de ar, resultando em fortes inversões de temperatura, conhecidas como inversões dos alísios. Esse processo limita o mecanismo convectivo e, dessa maneira, a formação de nuvens, diminuindo a quantidade de chuva. O período de transição caracteriza-se pelo início do deslocamento da ZCIT no sentido norte, em abril, e sul, em novembro, gerando alta variação na altura da precipitação pluvial. Já nos meses de maio a outubro, o sistema de pressões desloca-se para o norte permitindo a entrada da ZCIT no Estado, caracterizada por uma intensa atividade 
convectiva, originando o período úmido (Uzcategui \& González, 2000; D’Ambrosio, 2003).

Considerando separadamente esses três períodos, a análise de agrupamento permitiu definir quatro regiões homogêneas de localidades com precipitação mensal similar para os períodos seco e úmido, e três no período de transição (Figuras 2, 3 e 4). O mesmo critério utilizado na determinação do número de períodos foi adotado para o número de regiões, considerando também a distribuição espacial das localidades.

No período seco, a região homogênea S3 agrupou locais com menores índices de chuva, em que todos os meses tiveram precipitação inferior a $60 \mathrm{~mm}$, com valores médios entre 33,1 mm, Lobatera, e 43,3 mm, P. Batallon (Figura 2). As localidades H. V. Negro, P. Batallon, Pueblo Hondo e Sabana Grande encontramse na vertente "llanera" e com altitudes superiores a $2.000 \mathrm{~m}$. Na região S4, as localidades tiveram os maiores valores, com um mínimo de 88,1 mm, em Puente Salon, e máximo de 170,2 mm, em Táchira. Todas as localidades dessa região encontram-se entre a base e a encosta da cordilheira, com altitudes variando de 340 m,
Táchira, a 1.830 m, San Vicente. Isso evidencia o sistema característico da região, no qual a chuva decorre de mecanismos convectivos orográficos que forçam a ascendência de massas de ar úmido provenientes da vertente "llanera", em Uribante, Santa Fé, Puente Salon e San Vicente, e da "lacustrina", em Táchira e Colón (Uzcategui \& González, 2000).

Nas regiões homogêneas S1 e S2, o período seco se restringe de janeiro a março, sendo em dezembro os valores da altura de chuva superiores a $65 \mathrm{~mm}$. Porém, na região S1, esses valores foram ligeiramente inferiores (de 66,7 mm, em Los Laureles, a 83,8 mm, em Las Adjuntas) aos da S2 (entre 83,9 mm, em Zorca, e 112,2 mm, em La Cope), sendo Las Dantas a exceção, com $87,0 \mathrm{~mm}$. A classe S1 encontra-se no extremo sudoeste do Estado, onde a chuva é influenciada na escala regional pelos sistemas provenientes da Colômbia.

Nos meses de transição, a região T3 mostrou valores de precipitação superiores aos das regiões T1 e T2 nessa ordem, variando entre $164,3 \mathrm{~mm}$, em La Cope, e 343,8 mm, em Uribante (Figura 3). As localidades dessa região foram similares às da $\mathrm{S} 4$, assumindo que são

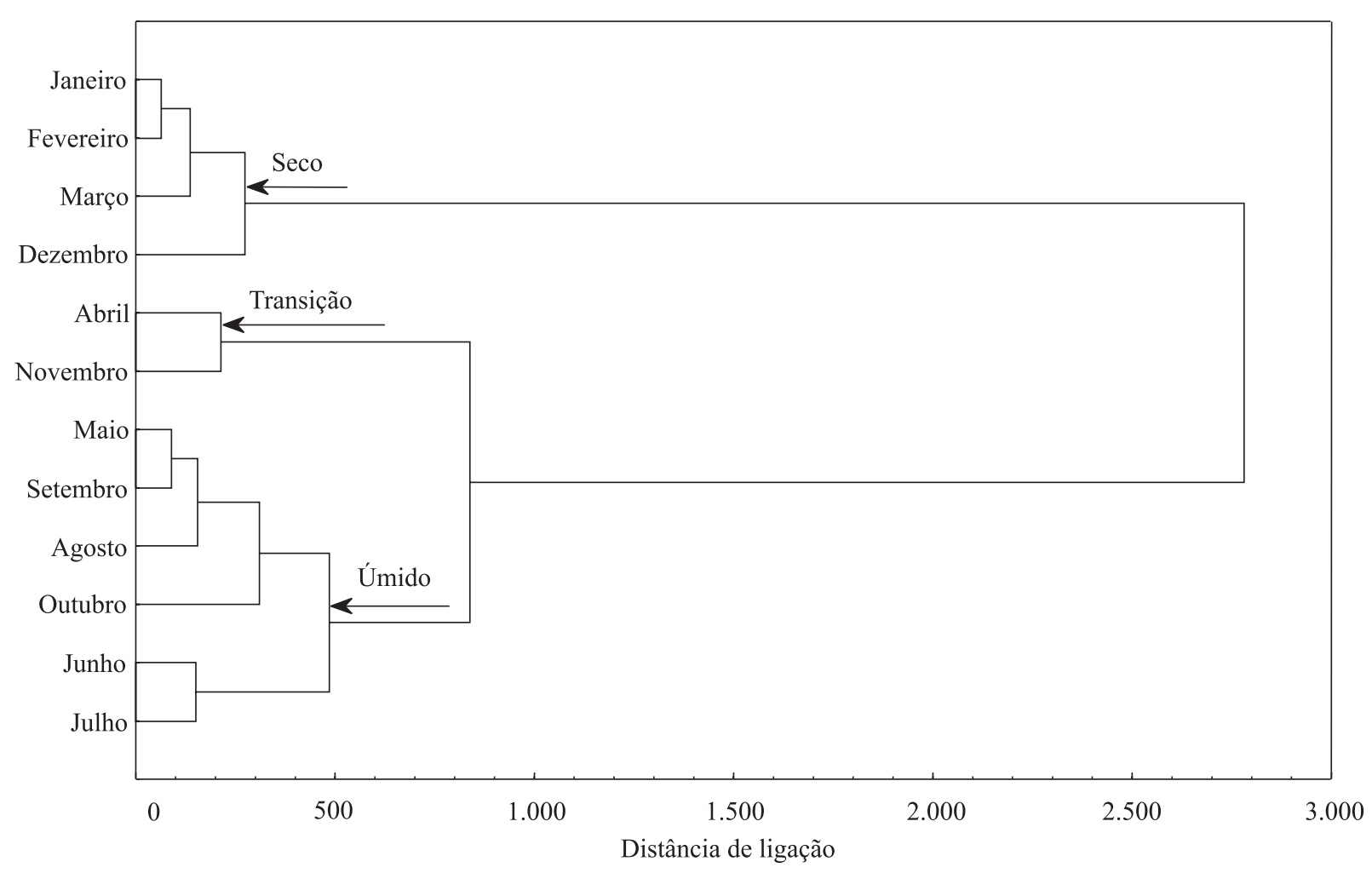

Figura 1. Dendrograma dos períodos de precipitação pluvial mensal homogênea das estações climatológicas do Estado de Táchira, Venezuela pelo método de agrupamento de Ward. 
influenciadas pelos mesmos sistemas locais. Contudo inclui-se em T3, Doradas, La Cope e Las Coloradas, evidenciando ainda, nesses meses, o efeito da ZCIT. Espacialmente a região T2 não mostrou continuidade, distribuindo-se em três regiões do estado: no nordeste, nas localidades H. V. Negro, P. Batallon, Pueblo Hondo e Sabana Grande; no sudoeste, em Rubio e San Vicente; e no oeste, em Ureña, Lobatera e Zorca. Na região T2, os valores de chuva foram registrados entre $83,6 \mathrm{~mm}$, em Rubio Marn, e 132,7 mm, em San Vicente. A precipitação na região $\mathrm{T} 1$ variou de um mínimo de 111,2 mm, em El Recreo, a um máximo de 129,4 mm na localidade de Delicias.
As quatro regiões homogêneas formadas no período úmido evidenciam a variação crescente da chuva na direção sudeste (Figura 4). Os locais com regime de precipitação bimodal (região U2) foram agrupados numa única região mais ao noroeste da cordilheira, com precipitação média entre 58,1 mm, Ureña, e $80,2 \mathrm{~mm}$, Las Adjuntas, seguido de U1 situada na encosta e variação entre 95,7 mm, em El Recreo, e 134,7 mm, em Bramón. Em oposição, os locais que formam as regiões U3 e U4 apresentam regime unimodal e encontram-se ao sudeste da cordilheira. As localidades de U3 estão mais distantes da cordilheira e com os

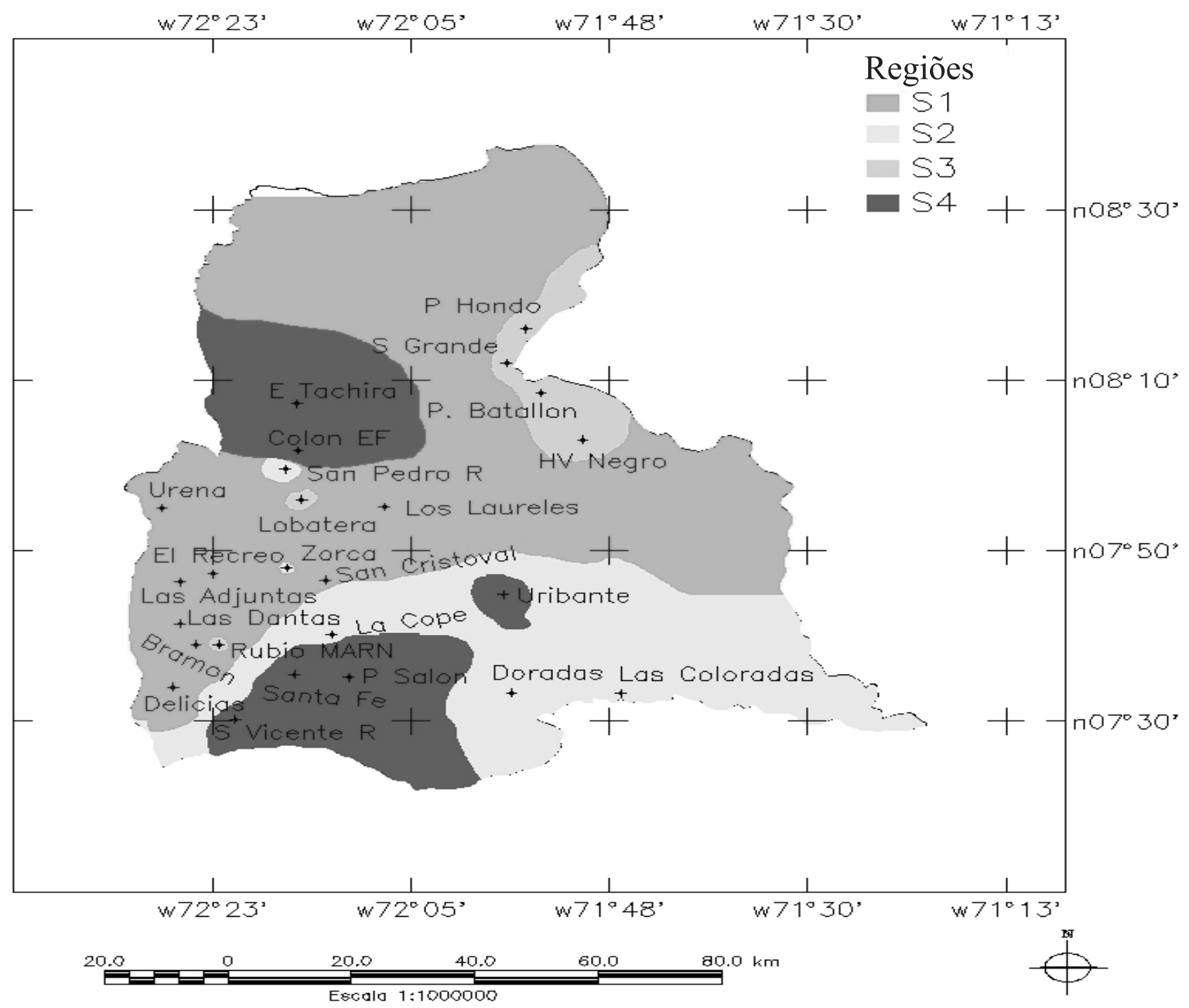

Figura 2. Distribuição espacial das regiões homogêneas para o período seco (S), pelo método de agrupamento de Ward, baseado na precipitação pluvial mensal das estações climatológicas do Estado de Táchira, Venezuela. 
maiores valores, variando de 327,1 mm, Las Coloradas, a 486,2 mm, Uribante, enquanto em U4, a variação foi entre 176,8 mm, H. V. Negro, e 283,2 mm, La Cope.
No período seco, a distribuição exponencial predominou, ajustando-se a 36\% dos locais, em março, e a 56\% em fevereiro (Tabela 1). As localidades da região S4

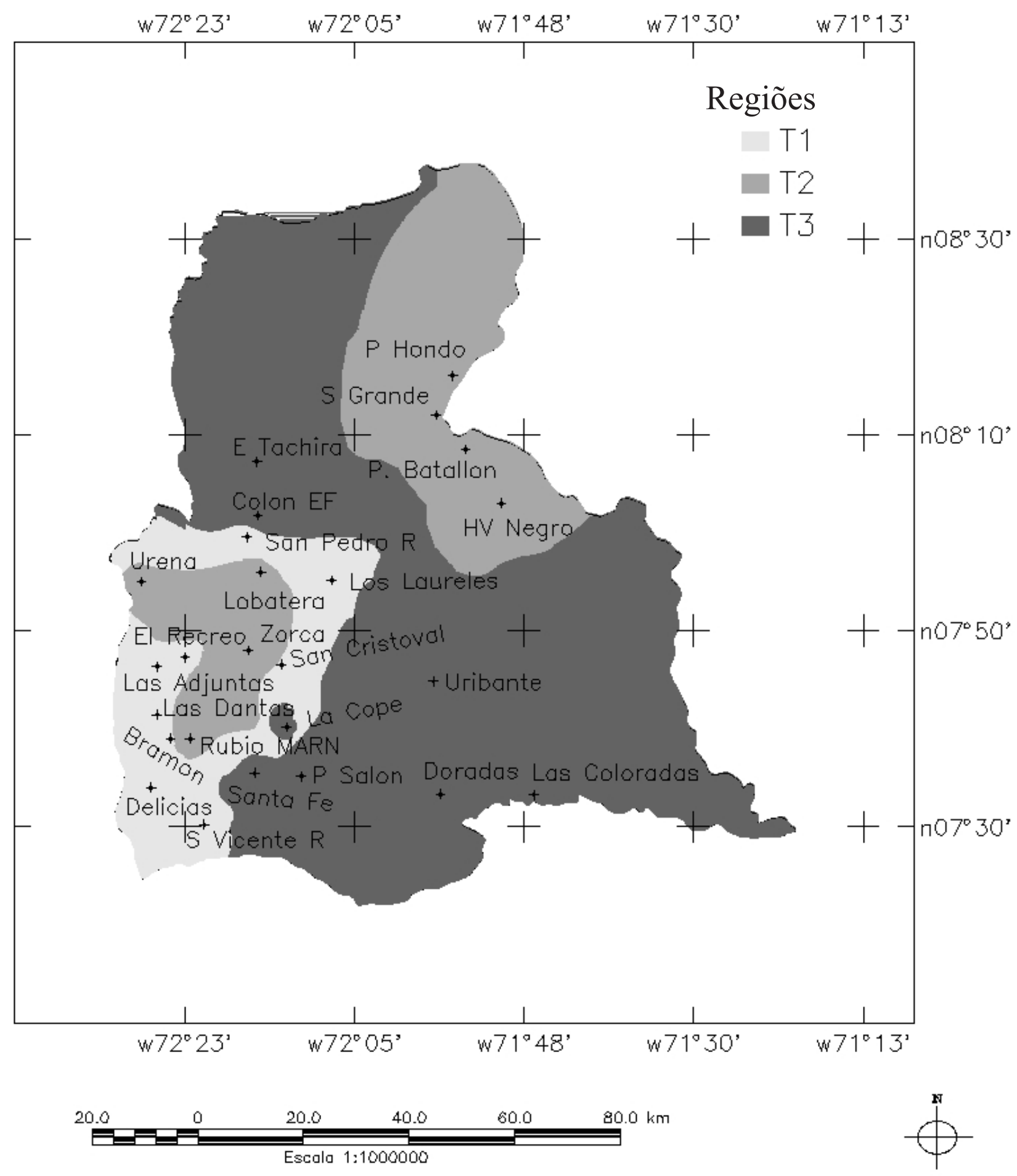

Figura 3. Distribuição espacial das regiões homogêneas para o período de transição (T), pelo método de agrupamento de Ward, baseado na precipitação pluvial mensal das estações climatológicas do Estado de Táchira, Venezuela. 


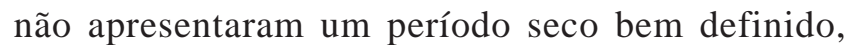

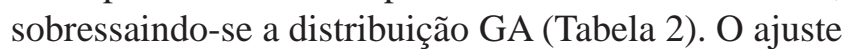

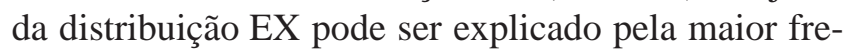

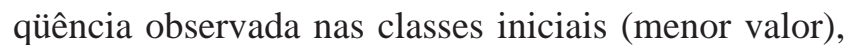

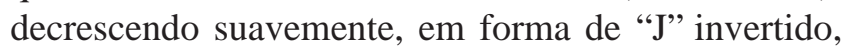

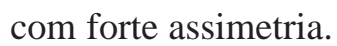

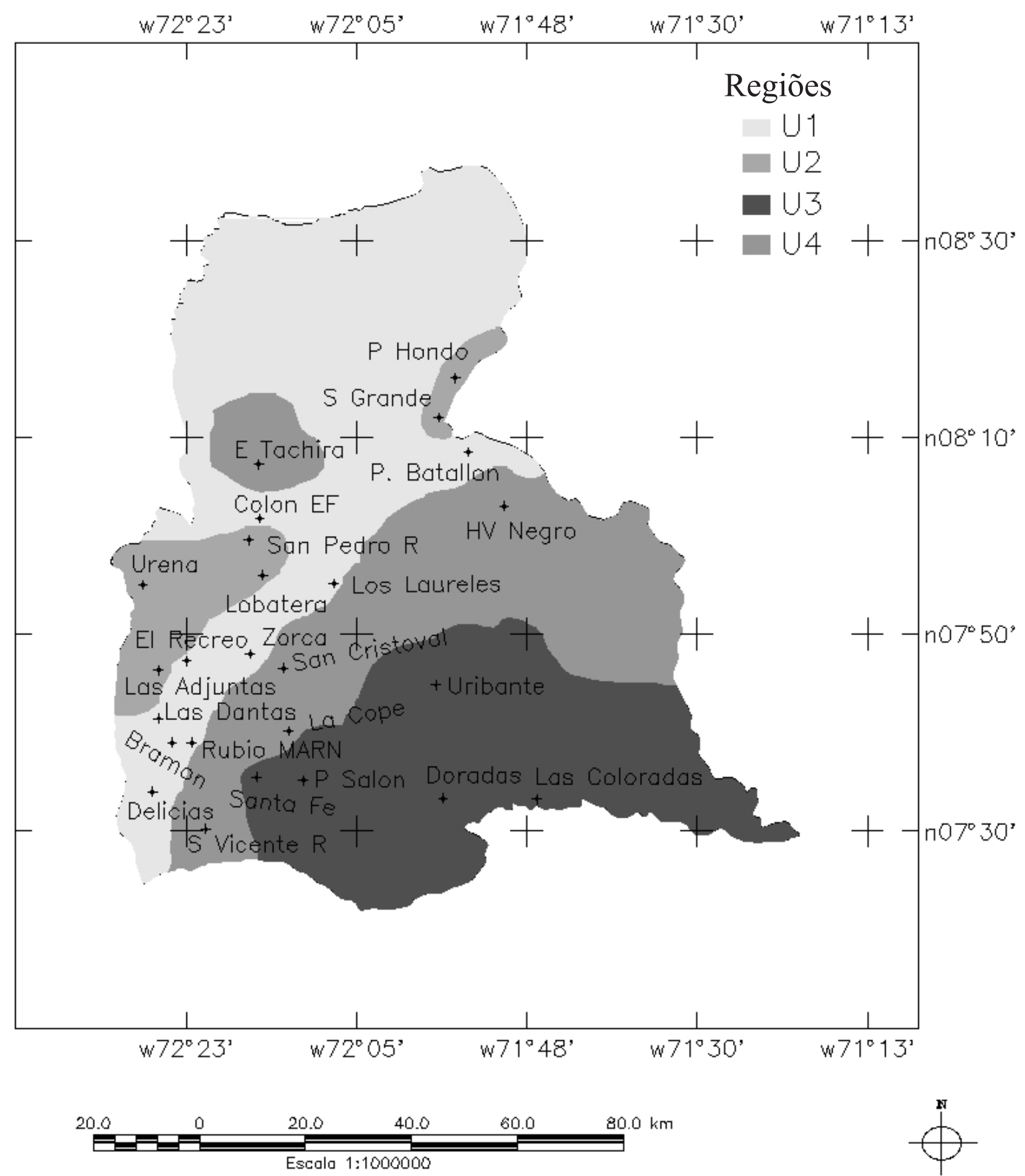

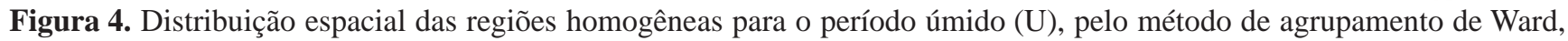

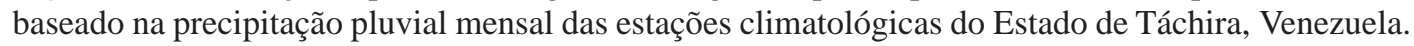


Resultado similar foi encontrado por Catalunha et al. (2002), os quais concluíram que, na estimativa da probabilidade da precipitação mensal, a distribuição EX foi a que apresentou melhores resultados nos meses secos, em Minas Gerais, Brasil.

Em janeiro e dezembro, a predominância da EX foi seguida pela gama, com ajuste de $36 \%$ em cada um deles. Isso se deve em parte à distribuição exponencial ser um caso particular da gama, com seu parâmetro de forma $(\alpha)$ igual a 1 (Frizzone, 1979). Nas localidades com melhor ajuste à distribuição gama em janeiro, $\alpha$ mostrou valores próximos de 1 , entre 0,67 e 1,74 . Essa mesma tendência foi observada para dezembro, porém a amplitude de $\alpha$ foi superior e sempre maior que 1 $(1,03 \leq \alpha \leq 2,33)$, com exceção de Uribante, em que a foi igual a 4,33 .

A distribuição das classes de freqüências dessas localidades mostrou um decréscimo das iniciais para as finais, sem o formato de "J" invertido suave característico da EX. Algumas dessas localidades apresentaram valores das classes intermediárias próximos das iniciais e em outras, as intermediárias com valores menores ou maiores que as suas adjacentes. Duan et al. (1998) comentam que a EX, por possuir apenas um parâmetro, não se mostra eficiente em estimar variações das classes de freqüências, ao contrário, a gama a dois

Tabela 1. Porcentagem de aderências das funções de distribuição de probabilidade à precipitação pluvial mensal das estações climatológicas do Estado de Táchira, Venezuela, utilizadas com base no teste de qui-quadrado $\left(\chi^{2}\right)$.

\begin{tabular}{|c|c|c|c|c|c|c|c|c|c|c|c|c|}
\hline \multirow{2}{*}{$\begin{array}{l}\text { Função de distribuição } \\
\text { de probabilidade }\end{array}$} & \multicolumn{12}{|c|}{ Porcentagem de aderência } \\
\hline & Jan. & Fev. & Mar. & Abr. & Maio & Jun. & Jul. & Ago. & Set. & Out. & Nov. & Dez. \\
\hline Exponencial & 44 & 56 & 36 & 28 & 8 & 8 & 0 & 0 & 0 & 4 & 4 & 40 \\
\hline Gama & 36 & 20 & 24 & 44 & 16 & 20 & 16 & 36 & 12 & 24 & 24 & 36 \\
\hline Gumbel & 0 & 4 & 8 & 12 & 4 & 20 & 8 & 28 & 16 & 12 & 24 & 4 \\
\hline Normal & 8 & 16 & 16 & 12 & 68 & 44 & 60 & 20 & 48 & 48 & 36 & 12 \\
\hline Log-normal & 0 & 0 & 8 & 0 & 0 & 0 & 0 & 0 & 0 & 4 & 8 & 4 \\
\hline Weibull & 4 & 4 & 8 & 0 & 4 & 0 & 4 & 8 & 8 & 0 & 0 & 0 \\
\hline Significativo $^{(1)}$ & 8 & 0 & 0 & 4 & 0 & 8 & 12 & 8 & 16 & 8 & 4 & 4 \\
\hline
\end{tabular}

(1)Ho significativo a 5\%: nenhuma das distribuições avaliadas se ajustou aos dados amostrais.

Tabela 2. Funções de distribuições de probabilidade que melhor se ajustaram à precipitação pluvial mensal das estações climatológicas do Estado de Táchira, Venezuela, utilizadas com base no teste de qui-quadrado $\left(\chi^{2}\right)$.

\begin{tabular}{|c|c|c|c|c|c|c|c|c|c|c|c|c|}
\hline \multirow[t]{2}{*}{ Estação climatológica } & \multicolumn{12}{|c|}{ Distribuição de probabilidade ${ }^{(1)}$} \\
\hline & Jan. & Fev. & Mar. & Abr. & Maio & Jun. & Jul. & Ago. & Set. & Out. & Nov. & Dez. \\
\hline Bramón & GA & WE & GA & GA & GA & $\mathrm{GU}$ & $\mathrm{NO}$ & GA & $\mathrm{GU}$ & GU & GA & GA \\
\hline Colon EF & GA & NO & WE & GA & NO & GA & NO & GA & NO & NO & GU & GA \\
\hline Delicias & EX & NO & NO & EX & NO & GA & GA & GU & NO & $\mathrm{LN}$ & GA & GA \\
\hline Doradas & $*$ & EX & EX & GA & NO & NO & & GU & NO & GA & NO & GA \\
\hline E1 Recreo & GA & NO & $\mathrm{LN}$ & GA & NO & NO & $\mathrm{NO}$ & NO & $*$ & ${ }^{*}$ & NO & GA \\
\hline Táchira & NO & GA & WE & GA & $\mathrm{NO}$ & $\mathrm{NO}$ & $\mathrm{NO}$ & GU & $\mathrm{NO}$ & NO & GA & $\mathrm{EX}$ \\
\hline H. V. Negro & NO & EX & GA & NO & GA & NO & GA & * & NO & * & GA & $\mathrm{EX}$ \\
\hline La COPE & WE & EX & $\mathrm{LN}$ & NO & $\mathrm{NO}$ & NO & GA & GA & & NO & GU & $*$ \\
\hline Las Adjuntas & EX & EX & $\mathrm{EX}$ & EX & $\mathrm{EX}$ & GU & NO & GU & GA & EX & EX & $\mathrm{EX}$ \\
\hline Las Coloradas & $\mathrm{EX}$ & $\mathrm{EX}$ & GA & $\mathrm{EX}$ & GA & NO & NO & NO & NO & NO & NO & NO \\
\hline Las Dantas & GA & EX & EX & EX & $\mathrm{EX}$ & GU & NO & GA & & GA & GU & GA \\
\hline Lobatera & EX & GA & $\mathrm{EX}$ & GU & NO & GU & GU & WE & WE & NO & NO & $\mathrm{EX}$ \\
\hline Los Laureles & GA & NO & $\mathrm{EX}$ & GA & $\mathrm{NO}$ & GA & $*$ & GA & GA & GU & GA & $\mathrm{EX}$ \\
\hline P. Batallon & GA & EX & $\mathrm{EX}$ & GA & NO & NO & NO & NO & NO & GA & NO & GA \\
\hline Pueblo Hondo & GA & EX & GA & EX & NO & EX & * & NO & GA & GA & LN & GA \\
\hline Puente Salon & EX & GA & GA & GU & $\mathrm{NO}$ & GU & GA & GA & $\mathrm{NO}$ & NO & NO & GU \\
\hline Rubio Marn & EX & EX & GU & & GA & & $\mathrm{NO}$ & $\mathrm{GU}$ & $\mathrm{GU}$ & GU & NO & $\mathrm{EX}$ \\
\hline Sabana grande & $\mathrm{EX}$ & EX & $\mathrm{EX}$ & GA & NO & EX & NO & GA & GU & GA & NO & $\mathrm{EX}$ \\
\hline San CristovalEst. & $*$ & GA & NO & GA & NO & NO & NO & GU & NO & NO & GU & $\mathrm{EX}$ \\
\hline San Pedro del Rio & EX & EX & NO & EX & NO & GA & NO & GA & ${ }^{*}$ & NO & NO & LN \\
\hline San Vicente R. & EX & GU & GA & $\mathrm{GU}$ & NO & NO & NO & GA & GU & GA & GU & NO \\
\hline Santa Fé & GA & GA & GU & NO & NO & GA & NO & GU & NO & NO & GA & NO \\
\hline Ureña & EX & $\mathrm{EX}$ & $\mathrm{EX}$ & EX & WE & NO & WE & WE & WE & NO & LN & $\mathrm{EX}$ \\
\hline Uribante - Presa & GA & $\mathrm{EX}$ & NO & GA & NO & $*$ & NO & NO & NO & NO & $*$ & GA \\
\hline Zorca & EX & $\mathrm{EX}$ & $\mathrm{EX}$ & GA & GU & $\mathrm{NO}$ & $\mathrm{GU}$ & & $\mathrm{NO}$ & NO & GU & $\mathrm{EX}$ \\
\hline
\end{tabular}

(1)EX: exponencial; GA: gama; GU: gumbel; NO: normal; LN: log normal; WE: Weibull. *Significativo a 5\%: nenhuma das distribuições avaliadas se ajustou aos dados amostrais. 
parâmetros é mais flexível, se ajustando bem a essas localidades. Catalunha et al. (2002) também observaram essa ineficiência da EX.

A distribuição normal predominou no período úmido, com exceção de agosto, ajustando-se de 44 a 68\% das localidades em junho e maio, respectivamente. As outras distribuições avaliadas apresentaram aderência inferior a $24 \%$ das localidades. Nesses meses, a maioria das localidades que se ajustaram à distribuição NO apresentaram alta simetria. Entretanto, as que possuem meses secos dentro do período úmido (região U2) não apresentaram tendência definida. $\mathrm{O}$ ajuste variou entre as distribuições NO, WE, GA, GU e EX nessa ordem, com quatro aderências para a NO e WE, três para GA e GU e uma para EX.

Nesse sentido, Frizzone (1979) cita que a NO se ajusta bem aos valores de precipitação semanal, mensal e sazonal que não apresentem muitos períodos secos. Igualmente, Catalunha et al. (2002) observaram que, no período úmido, as distribuições GA e NO, nessa ordem, mostraram maior número de ajustes.

Tais resultados, para fins de planejamento, facilitam a estimação das precipitações nessas localidades, visto que a distribuição normal necessita de apenas dois parâmetros de fácil determinação, isto é, a média e o desvio padrão.

Quanto ao mês de agosto, as distribuições GA, GU e NO apresentaram desempenho similares, sobressaindose entre as demais, com ajuste em 36,28 e $20 \%$ das localidades, respectivamente. Por sua vez, nas regiões U1, U3 e U4 predominou a GA com melhor ajuste.

No período de transição, destaca-se a distribuição GA, em abril (44\%), seguida pela EX (24\%). Essa variação pode ser atribuída por ser este um mês de transição entre seco e úmido em $76 \%$ das estações. No caso da EX, houve maior freqüência nos valores menores de chuva, mostrando o histograma característico dessa distribuição. Já na GA, o coeficiente de assimetria é mais próximo de zero. Em novembro, predominou a NO com $36 \%$ de ajuste e as distribuições GA e GU com $24 \%$.

Independentemente do período, as distribuições log normal e Weibull não apresentaram ajuste satisfatório. A LN mostrou aderências apenas em março e de outubro a dezembro, variando entre 4 e $8 \%$ enquanto para WE, não foi observada aderência de outubro a dezembro e em abril e junho. Alta taxa de rejeição da LN para a precipitação mensal foi também observada por Ali et al. (2000) e Catalunha et al. (2002).

\section{Conclusões}

1. A variação sazonal da precipitação pluvial no Estado de Táchira apresenta três períodos, estatisticamente definidos como seco, de transição e úmido.

2. Os períodos seco e úmido apresentam quatro regiões homogêneas de localidades com precipitação pluvial mensal similar e o período de transição apresenta três.

3. No período seco, a distribuição de probabilidade recomendada para as estimativas mensais é a exponencial, com exceção da região homogênea com os maiores valores da precipitação, onde a gama se sobressai.

4. No período úmido, em todas as regiões, o melhor desempenho é da distribuição normal, com exceção de agosto, em que a gama predomina.

5. Nos meses de transição destacam-se as distribuições gama, em abril, e normal, em novembro.

\section{Agradecimentos}

Ao Prof. Dr. Gilberto Sediyama, do Dep. de Engenharia Agrícola da Universidade Federal de Viçosa, e ao Dr. Márcio Catalunha, por disponibilizarem o software Estatística Aplicada a Climatologia (EClima).

\section{Referências}

ALI, A.; ABTEW, W.; VAN HORN, S.; KHANAL, N. Temporal and spatial characterization of rainfall over Central and South Florida. Journal of the American Water Resources Association, v.36, p.833-848, 2000.

ASSIS, F.N. de; ARRUDA, H.V. de; PEREIRA, A.R. Aplicações de estatística a climatologia: teoria e prática. Pelotas: Universitária, 1996. 161p.

BACK, Á.J. Seleção de distribuição de probabilidade para chuvas diárias extremas do Estado de Santa Catarina. Revista Brasileira de Meteorologia, v.16, p.211-222, 2001.

CARGNELUTTI FILHO, A.; MATZENAUER, R.; TRINDADE, J.K. da. Ajustes de funções de distribuição de probabilidade à radiação solar global no Estado do Rio Grande do Sul. Pesquisa Agropecuária Brasileira, v.39, p.1157-1166, 2004.

CATALUNHA, M.J.; SEDIYAMA, G.C.; LEAL, B.G.; SOARES, C.P.B.; RIBEIRO, A. Aplicação de cinco funções densidade de probabilidade a séries de precipitação pluvial no Estado de Minas Gerais. Revista Brasileira de Agrometeorologia, v.10, p.153-162, 2002.

CHESSA, P.A.; CESARI, D.; DELITALA, A.M.S. Mesoscale precipitation and temperature regimes in Sardinia (Italy) and their related synoptic circulation. Theoretical and Applied Climatology, v.63, p.195-221, 1999. 
D’Ambrosio, S. El Clima. Disponível em: <http:// www.monografias.com/trabajos4/elclima/elclima.html>. Acesso em: 20 jun. 2003.

DUAN, J.; SELKER, J.; GRANT, G.E. Evaluation of probability density functions in precipitation models for the Pacific Northwest. Journal of the American Water Resources Association, v.34, p.617-627, 1998.

EVERITT, B.S.; DUNN, G. Applied multivariate analysis. London: Edward Arnold, 1991. 400p.

FRIZZONE, J.A. Análise de cinco modelos para cálculo da distribuição e freqüência de precipitações na região de Viçosa, MG. 1979. 100p. Dissertação (Mestrado) - Universidade Federal de Viçosa, Viçosa.

HASTINGS, N.A.J.; PEACOCK, J.B. Statistical distributions: a handbook for students and practitioners. New York: J. Wiley, 1975. 130p.

MELlO, M.H.A.; ARRUDA, H.V.; ORTOLANI, A.A. Probabilidade de ocorrência de totais pluviais máximos horários em Campinas - São Paulo. Revista do Instituto Geológico, v.15, p.59-67, 1994.
RIBEIRO, A.M. de A.; LUNARDI, D.M.C. A precipitação mensal provável para Londrina PR, através da função gama. Energia na Agricultura, v.12, p.37-44, 1997.

SILVA, F.A.M. da; ASSAD, E.D. Análise temporal do potencial hídrico climático do Estado de Goiás. In: ASSAD, E.D.; SANO, E.E. (Org.). Sistema de informações geográficas: aplicações na agricultura. 2.ed. Brasília: Embrapa SPI, 1998. p.273-309.

THOM, H.C.S. A note on the gamma distribution. Monthly Weather Review, v.86, p.117-122, 1958.

UNAL, Y.; KINDAP, T.; KARACA, M. Redefining the climate zones of Turkey using cluster analysis. International Journal of Climatology, v.23, p.1045-1055, 2003.

UZCÁTEGUI, A.; GONZÁLEZ, M.G. El clima de Venezuela: unidade IV; climas de Venezuela. Santa Bárbara de Zulia: Universidad Sur del Lago, 2000. Disponível em: <http://www.unesur.edu.ve/ unidades/gencon/contclimatologia.html>. Acesso em: 20 mar. 2003.

WARD, J.H. Hierarchical grouping to optimize an objective function. Journal of the American Statistical Association, v.58, p.236-244, 1963.

Recebido em 29 de julho de 2004 e aprovado em 25 de julho de 2005 